\title{
Detection of static moving objects using multiple nonparametric background models
}

\author{
Raquel Martínez, Carlos Cuevas, Daniel Berjón and Narciso García
}

\begin{abstract}
Detection of moving objects remaining static is a fundamental step in many computer vision applications, since it allows to identify potentially dangerous situations (abandoned objects) and people temporally static. Here, we propose a strategy to efficiently detect such static moving objects, which is based on three nonparametric background models (long term, medium term and short term) to detect moving objects and a novel Finite State Machine to identify when a moving object becomes static.
\end{abstract}

Keywords-detection; static moving object; nonparametric modeling; finite state machine

\section{INTRODUCTION}

Detection of suspicious behavior has become a task of interest in video surveillance along the last years. Consequently, several strategies focused on identifying abandoned objects can be found in the recent literature [1]. Most of these algorithms are based on analyses at object-level and are focused exclusively on the detection of abandoned objects [2]. However, they fail in sequences with people that stop walking (in these situations people tend to move the upper body but not the legs) and, additionally, they establish some criteria (e.g. minimum object size) that make it difficult to detect small objects. To solve these problems, some approaches perform the classification at pixel-level [3] [4], including an initial foreground detection step that is commonly based on the application of a parametric background modeling using Mixtures of Gaussians (MoG). Nevertheless, they fail in sequences with very dynamic backgrounds where parametric methods are not able to correctly model the pixel variations [5] and, moreover, they depend on many parameters, which drastically decreases their usability.

We propose a high-quality strategy to detect moving objects that remain partially or totally static. To detect moving objects, we apply a nonparametric background model with 3 different temporal buffers. The detection results are introduced into a Finite State Machine (FSM) to identify when a moving object becomes static and also when a static moving object moves again. The obtained results have shown that the proposed algorithm not only improves the quality of previous approaches but it also avoids the manual selection of parameters.

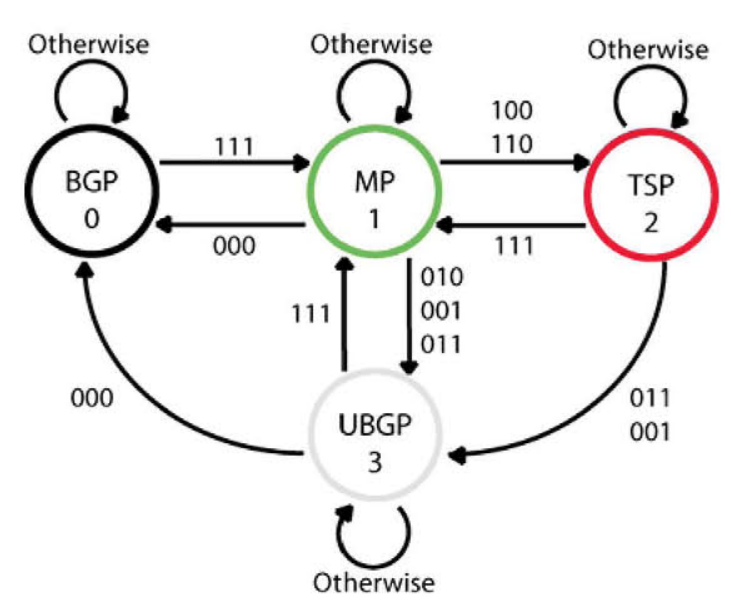

Fig. 1. Proposed FSM. Black: Background pixels. Green: Moving pixels. Red: Temporally static pixels . Gray: Uncovered background pixels.

\section{PROPOSED STRATEGY}

\section{A. Background modeling}

Let $\mathbf{x}^{n}$ be a pixel in the current image, at time $n$, defined by its RGB color components $\left(R^{n}, G^{n}, B^{n}\right)$. The probability of such pixel belonging to the foreground, $\operatorname{Pr}\left(\phi \mid \mathbf{x}^{n}\right)$, can be evaluated [5] using Bayes' theorem,

$$
\operatorname{Pr}\left(\beta \mid \mathbf{x}^{n}\right)=1 /\left(1+p\left(\mathbf{x}^{n} \mid \beta\right)\right),
$$

where $p\left(\mathbf{x}^{n} \mid \beta\right)$ is the probability density function (pdf) that $\mathbf{x}^{n}$ belongs to the sequence background, $\beta$, which can be nonparametrically estimated from a set of $T_{\beta}$ reference samples, $\left\{\mathbf{x}_{\beta}^{i}\right\}$, and using Gaussian kernels as

$$
p\left(\mathbf{x}^{n} \mid \beta\right)=K_{w} \sum_{i=1}^{T_{\beta}} \prod_{j=1}^{3} \exp \left(-\frac{\left(x^{n}(j)-x_{\beta}^{i}(j)\right)^{2}}{2 \Sigma(j, j)}\right),
$$

where $K_{w}$ is a normalization factor, and $\sum$ is the covariance matrix determining the width of the Gaussian kernels. 

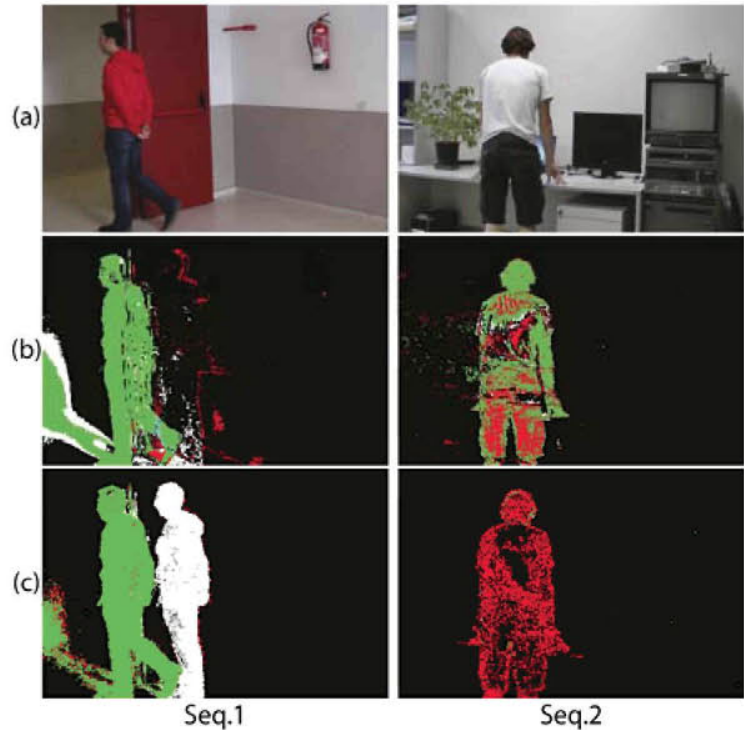

Fig. 2. (a) Original images. (b) Results with the strategy in [4]. (c) Results with the proposed strategy. Color notation: temporally static pixels in red, moving pixels in green, and uncovered background in white.

From these equations, we obtain 3 probabilities for each pixel to belong to the foreground, each of which is obtained by only changing the value of $T_{\beta}$. In this way, the largest $T_{\beta}$ value provides a long term detection, the medium $T_{\beta}$ value provides a medium term detection, and the shortest $T_{\beta}$ value provides a short term detection. Finally, the probabilities obtained for each current pixel, $\mathbf{x}^{n}$, are thresholded by 0.5 to obtain a binary vector, $\mathbf{F}=\left(F_{L} F_{M} F_{S}\right)$, whose elements correspond respectively to the long, medium and short term models.

\section{B. Finite State Machine}

The inputs of the proposed FSM, which is illustrated in Fig. 1 , are the binary vectors obtained in the previous stage. According to the current state for each pixel and these vectors, the proposed FSM classifies the pixels in 4 possible states:

(0) BGP: Background pixels. This is the initial state for every pixel, and it denotes that the pixel is part of the background. The pixel leaves this state when it is part of a moving region and, therefore, $\mathbf{F}=(1,1,1)$.

(1) MP: Moving pixels. A pixel in this state that no longer is part of a moving region will change to UBG (when $\mathbf{F}=(0, x, x)$, excluding $\mathbf{F}=(0,0,0))$. If the pixel is rapidly absorbed by the three models, $F=(0,0,0)$, it will change to $B G$, whereas if it is absorbed only by the short and medium term models $(\mathbf{F}=(1, \mathrm{x}, 0))$, it will change to TSP.

(2) TSP: Temporally static pixels. A pixel in this state will change to UBG if it is part of a static object that starts moving $(\mathbf{F}=(0,0,1)$ or $\mathbf{F}=(0,1,1))$, and to MP when it is covered by other moving object or other values from the stopped moving object.

(3) UBGP: Uncovered background pixels (new background pixels corresponding to stopped moving objects). To exit this state the pixel has to be regarded as background by the 3 models $\mathbf{F}=(0,0,0)$, or as foreground by the 3 models, $\mathbf{F}=(1,1,1)$, if a moving region is overlapping.

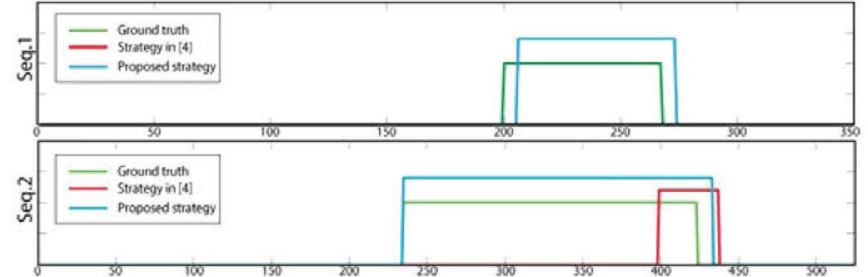

Fig. 3. Detected events for the sequences illustrated in Fig. 2.

\section{RESULTS}

The proposed strategy has been tested on several sequences from LASIESTA database [6]. The obtained results have been compared to those provided by the method in [4], which is an improved version of the algorithm proposed in [3]. This method is based on a parametric MoG-based approach to model the background and it also uses a FSM at pixel-level to determine when moving objects become static.

Figure 2 illustrates some representative results obtained for two sequences with moving objects that remain temporally static. The colors in these results correspond to those used in Fig. 1 to represent the states of the FSM. It can be noted that the proposed strategy classifies the pixels more accurately than [4]. Moreover, since we use a nonparametric algorithm, we are able to correctly model complex background variations (e.g. the changes in the plant in Seq. 2), whereas the MoG-based detection proposed in [4] leads to many false detections. Finally, Fig. 3 shows the detected events along the two sequences in Fig. 2. It can be seen that our strategy is very fast detecting static moving objects. However, the method in [4] is much slower and it does not work properly with objects remaining static very short periods of time (e.g. in Seq. 1).

\section{CONCLUSIONS}

We have proposed a high-quality strategy for detecting static moving objects in video sequences. Using a nonparametric background modeling with different temporal buffer sizes, moving objects are robustly detected at long, medium and short term. The detection results are introduced into a FSM that quickly classify the pixels as belonging to static moving objects. The obtained results have shown that the proposed method greatly improves the quality of previous approaches.

\section{REFERENCES}

[1] A. Bayona, J. SanMiguel and J. Martinez, "Stationary foreground detection using background subtraction and temporal difference in video surveillance”, IEEE Int. Conf. Image Procesing, pp. 4657-4660, 2010.

[2] L. Chang, H. Zhao, S. Zhai, Y. Ma and H. Liu, "Robust Abandoned Object Detection and Analysis Based on Online Learning", IEEE Int. Conf. Robotics and Biomimetics, pp. 940-945, 2013.

[3] F. Porikli, Y. Ivanov, and T. Haga, "Robust abandoned object detection using dual foregrounds", EURASIP Journal on Advances in Signal Processing, pp. 197875:1-11, 2008.

[4] R.H. Evangelio and T. Sikora, "Static object detection based on a dual background model and a finite-state machine", EURASIP Journal on Image and Video Processing, pp. 858502:1-11, 2011.

[5] C. Cuevas, N. García, "Efficient Moving Object Detection for Lightweight Applications on Smart Cameras", IEEE Trans. Circuits and Systems for Video Technology, vol. 23, no. 1, pp. 1-14, Jan. 2013.

[6] http://www.gti.ssr.upm.es/data/ 